\title{
Surface Atomic Arrangement Visualization via Reference-Atom-Specific Holography
}

\author{
Huasheng Wu, ${ }^{1}$ Shihong Xu, ${ }^{1}$ Simon Ma, ${ }^{1}$ W. P. Lau, ${ }^{1}$ M. H. Xie, ${ }^{1}$ and S. Y. Tong ${ }^{2}$ \\ ${ }^{1}$ Department of Physics and HKU-CAS Joint Laboratory on New Materials, The University of Hong Kong, Hong Kong \\ ${ }^{2}$ Department of Physics and Materials Science, City University of Hong Kong, Hong Kong
}

(Received 6 June 2002; published 31 October 2002)

\begin{abstract}
We demonstrate the direct reconstruction of 3D atomic images from measured low-energy electron diffraction (LEED) intensity spectra. A multiple-incident angle and multiple-energy integral are first applied to the spectra to obtain a map of interatomic vectors. From this map, a nonbulk interatomic vector is chosen that points to a desired reference atom. A second integral transformation, using the chosen interatomic vector as a filter, is applied to the LEED spectra to produce images of individual atoms in the vicinity of the selected reference atom. This two-step method overcomes the problem of multiple, nonequivalent reference atoms and is applicable to elemental or compound materials.
\end{abstract}

PACS numbers: 68.35.Bs, 07.79.-v, 61.14.Hg, 68.55.-a

We demonstrate here the implementation of a new method for direct visualization of surface structures from measurements of low-energy electron diffraction spectra. As a beam of coherent electrons is scattered by atoms in a solid, the back-scattered wavelets reaching the detector suffer different propagation delay phases, depending on the scattering paths taken. Thus, two wavelets each singly scattered by atoms located at $\vec{r}_{i}$ and $\vec{r}_{j}$, respectively, have a propagation delay phase difference of $\Phi_{j i}=\left(\vec{k}_{f}-\vec{k}_{i}\right) \cdot\left(\vec{r}_{j}-\vec{r}_{i}\right)$, where $\vec{k}_{i}$ and $\vec{k}_{f}$ are the incident and final wave vectors. Likewise, the propagation delay phases of higher scattering paths are known functions of wave vectors $\left(\vec{k}_{i}, \vec{k}_{f}\right)$ and atomic positions $\left(\vec{r}_{i}, \vec{r}_{j}\right.$, etc.). All real space visualization methods using wave front reconstruction employ the same principle: Integral transformation of the data to phase lock onto a particular class of propagation delay phase differences. A wellknown example is holography (using photons or electrons) in which the propagation delay phase differences between a reference wave and singly scattered object waves are selected [1], while phase differences of multiply scattered waves are filtered out [2-13]. A major difficulty shared by the methods cited in these references is that they cannot separate object images from nonequivalent reference atoms wherever there are multilayers of the same kind of atoms. The method described in this Letter overcomes difficulty by using a two tier integral transformation. First, a multiple-energy integral transformation is applied to select from the data all singlescattering delay phases. This technique forms a map of interatomic vector distances. From this map, a particular surface interatomic distance is chosen. A second transformation, using the chosen surface interatomic distance as a filter, is then applied to the data to produce images of individual atoms in the vicinity of the chosen reference atom. The new selective holographic atomic-referencepair (SHARP) transformation produces a direct visualization of the surface geometry at the local environment of the selected reference atom. We demonstrate here how the method works for the $\operatorname{Si}(111) R 30^{\circ}-(\sqrt{3} \times \sqrt{3})-\mathrm{Ga}$ system shown in Fig. 1(a).

The low-energy electron diffraction (LEED) experiments are performed in a $\mu$-metal chamber interconnected under ultrahigh vacuum conditions to other chambers, including a preparation chamber where sample cleaning is carried out. The $\mathrm{Si}(111)$ crystal is mounted and the surface oxide is removed by thermal flashing at $1200{ }^{\circ} \mathrm{C}$ below $1.0 \times 10^{-9}$ torr by passing an electric current through the sample. The clean $\mathrm{Si}(111)$ surface shows sharp $7 \times 7$ LEED spots with a low background. The $\operatorname{Si}(111)-(\sqrt{3} \times \sqrt{3}) R 30^{\circ}$-Ga surface is prepared by depositing $\mathrm{Ga}$ atoms on the $\mathrm{Si}(111)(7 \times 7)$ surface at room temperature, followed by annealing at $650{ }^{\circ} \mathrm{C}$ [14]. The Ga flux is generated by e-beam evaporation. LEED IV spectra in the energy range $50-400 \mathrm{eV}$ are measured. The spectra for nine incident angles $\left(\theta=0^{\circ}-39^{\circ}, \varphi=\right.$ $\left.0^{\circ}-60^{\circ}\right)$ and $80-150$ diffracted beams at each incident angle are collected, depending on the symmetry of the incident azimuth. The angle $\theta$ is measured with respect to the surface normal, and the angle $\varphi$ is measured with respect to the [101] direction. Over $2 \times 10^{5}$ independent intensity $(I)$ vs energy $(E)$ data points are collected. The $I V$ spectra of two diffracted beams are shown in Fig. 1(b).

To obtain a real space visualization of surface structure, we first apply a multiple-energy integral transform to the data to select single-scattering propagation delay phases between the scattered electron wavelets. The LEED intensity spectra contain all orders of multiple scattering events. Of these, the scattered wavelets from atoms located at $\vec{r}_{i}$ and $\vec{r}_{j}$ are given by

$$
\begin{aligned}
I & =\left|f_{i} e^{-i \vec{q} \cdot \vec{r}_{i}}+f_{j} e^{-i \vec{q} \cdot \vec{r}_{j}}+\cdots\right|^{2} \\
& =\left|f_{i}\right|^{2}+f_{i} f_{j}^{*} e^{i \vec{q} \cdot \vec{r}_{j i}}+\cdots+\text { c.c., }
\end{aligned}
$$

where $\vec{q}=\vec{k}_{f}-\vec{k}_{i}, \vec{r}_{j i}=\vec{r}_{j}-\vec{r}_{i}$, and $f_{i}, f_{j}$ are atomic scattering factors. By an integral transform of the intensity, and summing over $\hat{q}$ and $\vec{k}_{i}$, we obtain 

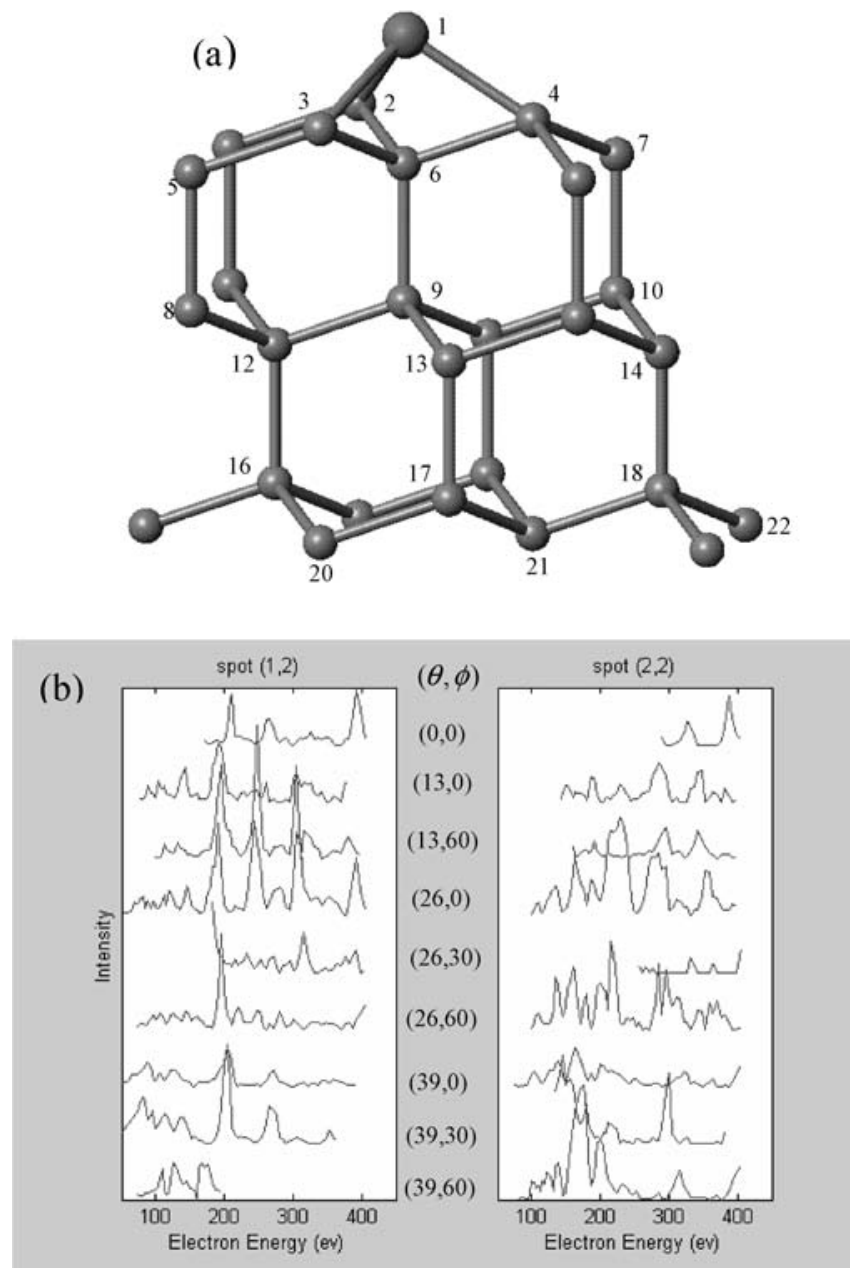

FIG. 1. (a) A representative unit of the $\operatorname{Si}(111)-(\sqrt{3} \times$ $\sqrt{3}) R 30^{\circ}$-Ga system where atoms are numbered as $\mathrm{Ga}(1)$, $\mathrm{Si}(2-22)$. Atoms $1,4,6,9$, etc., are in the (101) crystallographic plane. (b) LEED IV spectra of the $(1,2)$ and $(2,2)$ diffracted beams are shown for the nine incident angles collected in the data.

$$
P(\vec{r})=\left|\sum_{\vec{k}_{i}, \hat{q}} \int\left(\frac{I}{A}-1\right) e^{-i \vec{q} \cdot \vec{r}} d q\right|^{2} .
$$

Here $A$ is an average intensity over all energies and diffracted directions. The real space function $P(\vec{r})$ peaks at $\vec{r}=\vec{r}_{j i}$ [15]. At these points, the phase of the integration kernel $e^{-i \vec{q} \cdot \vec{r}}$ exactly cancels the propagation delay phase difference between two singly scattered wavelets from atoms located at $\vec{r}_{i}$ and $\vec{r}_{j}$. The sums over $\hat{q}$ and $\hat{k}_{i}$, using a backward cone of $60^{\circ}$, wash out contributions from higher orders. The peaks in $P(\vec{r})$ correspond to interatomic distances. Figure 2 shows $P(\vec{r})$ along the (101) crystallographic plane of the $\mathrm{Si}(111)$ $(\sqrt{3} \times \sqrt{3}) R 30^{\circ}-\mathrm{Ga}$ system. The map of $P(\vec{r})$ is not a direct visualization of surface structure because it is a map of interatomic distances. For $n$ atoms in a structure, there are $n(n-1) / 2$ interatomic distances in $P(\vec{r})$.

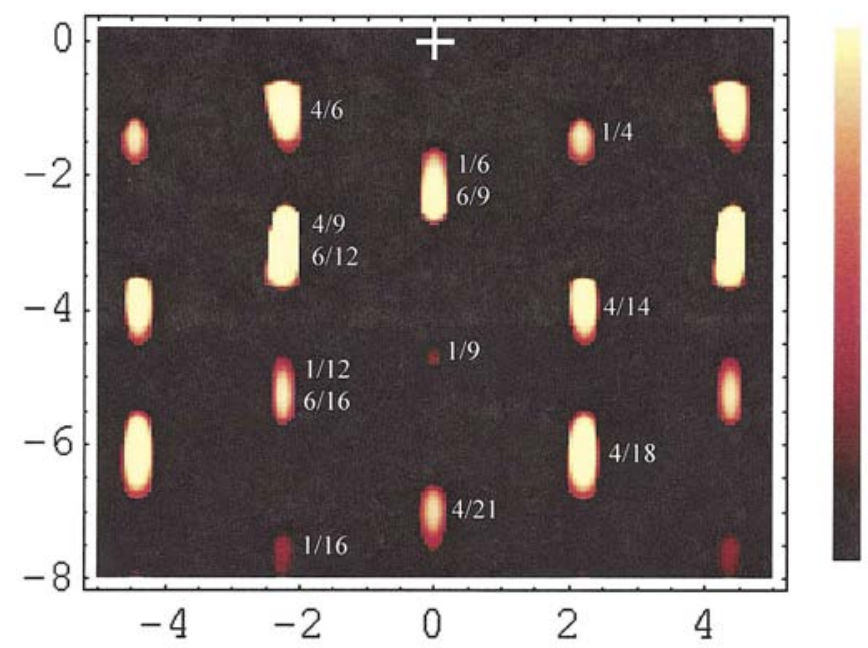

FIG. 2 (color). Map of interatomic distances obtained from the Patterson transformation of LEED data viewed in the (101) crystallographic plane for the $\operatorname{Si}(111)-(\sqrt{3} \times \sqrt{3}) R 30^{\circ}$-Ga system. The origin is marked by a cross. Each spot is labeled with two numbers corresponding to atoms numbered in Fig. 1; e.g., the spot $1 / 4$ represents the interatomic distance between atoms 1 and 4 .

Because $P(\vec{r})$ is equivalent to a surface Patterson map, we call the transformation kernel $e^{-i \vec{q} \cdot \vec{r}}$ the Patterson kernel [16-18].

The second and crucial step is to choose a particular atom as the reference atom and produce from the data a direct visualization of the surface structure relative to this reference atom. Since all interatomic distances $\vec{r}_{j i}$ of bulk $\mathrm{Si}(111)$ are known, it is easy to identify spots in $P(\vec{r})$ that do not correspond to a bulk Si-Si vector. Examples of nonbulk spots in Fig. 2 are 1/4, 1/9, etc. While any one of these spots may be chosen in the wave front reconstruction of the second step, the vector from the $1 / 4$ spot to the origin is selected because atom 4 is close to the surface. We choose this vector because contributions from shallow atoms are strong due to attenuation of the electronic wave inside a solid. Once the vector $\vec{r}_{14}$ is chosen, a second transformation is applied to the data. This transformation selects out propagation delay phases between singly scattered wavelets at atom 4 and doubly scattered wavelets whose first scattering is at atom 1 . The two scattering events are illustrated schematically in Fig. 3. The kernel of the second transformation, known as the selective SHARP kernel, is given by

$$
K_{s}=e^{i \vec{q} \cdot \vec{r}_{14}} e^{-i\left(k_{f} r-\vec{k}_{f} \cdot \vec{r}\right)} .
$$

The integral transform using the SHARP kernel is given by

$$
S(\vec{r})=\sum_{\vec{k}_{i}}\left|\sum_{\hat{k}_{f}} \int\left(\frac{I}{A}-1\right) e^{i \vec{q} \cdot \vec{r}_{14}} e^{-i\left(k_{f} r-\vec{k}_{f} \cdot \vec{r}\right)} d k_{f}\right|^{2} .
$$




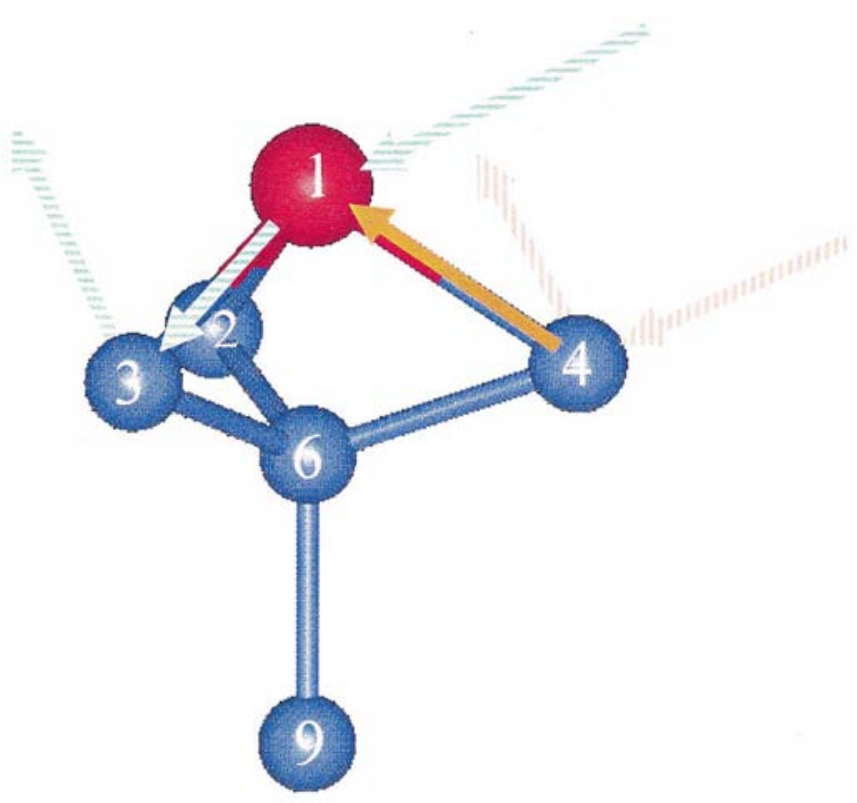

FIG. 3 (color). In the SHARP transformation, the vector distance from atom 4 to atom 1 (orange arrow) is chosen as a filter to exclusively form atomic images displayed relative to atom 1. An example of a phase delay between a singly scattered wavelet at atom 4 (light brown arrows) and doubly scattered wavelets first at atom 1 then at atom 3 (light green arrows) is shown.

The function $S(\vec{r})$ peaks at $\vec{r}=\vec{r}_{j}-\vec{r}_{1}$. In other words, the interatomic vector $\vec{r}_{14}$ in the SHARP kernel selects the reference atom at $\vec{r}_{1}$ from which all other atoms in the solid are measured.

To see why the SHARP transformation works, we write down the relative propagation delay phases from a singly scattered wavelet at atom 4 and a doubly scattered wavelet at atom 3 that uses atom 1 as the reference atom. The exponentials of this term are given by $e^{-i \vec{q} \cdot \vec{r}_{14}} e^{i\left(k_{f} r_{31}-\vec{k}_{f} \cdot \vec{r}_{31}\right)}$. One immediately recognizes that the SHARP kernel given in Eq. (3) is exactly conjugate to this term if $\vec{r}=\vec{r}_{31}$. It follows that the SHARP transformation phase locks onto this class of wavelets while the sums over $\hat{k}_{f}$ and $\vec{k}_{i}$ filter out other classes of wavelets. As a result, the SHARP integration seeks out the positions of all atoms measured exclusively relative to atom 1. In Fig. 4, we show $S(\vec{r})$ obtained from the SHARP transformation of the LEED data. Because $S(\vec{r})$ is a 3D image, we show it in different 2D cuts. Figure 4(a) shows the image in the (101) crystallographic plane and it contains atoms 4,6 , and 9 . The image is directly comparable to the arrangement of these atoms in the surface geometry shown in Fig. 1. Figure 4(b) contains the image of atoms 2, 3, and 4 in a horizontal plane and again is directly comparable to the surface geometry shown in Fig. 1. All atomic images have a 3D resolution better than $1 \AA$.
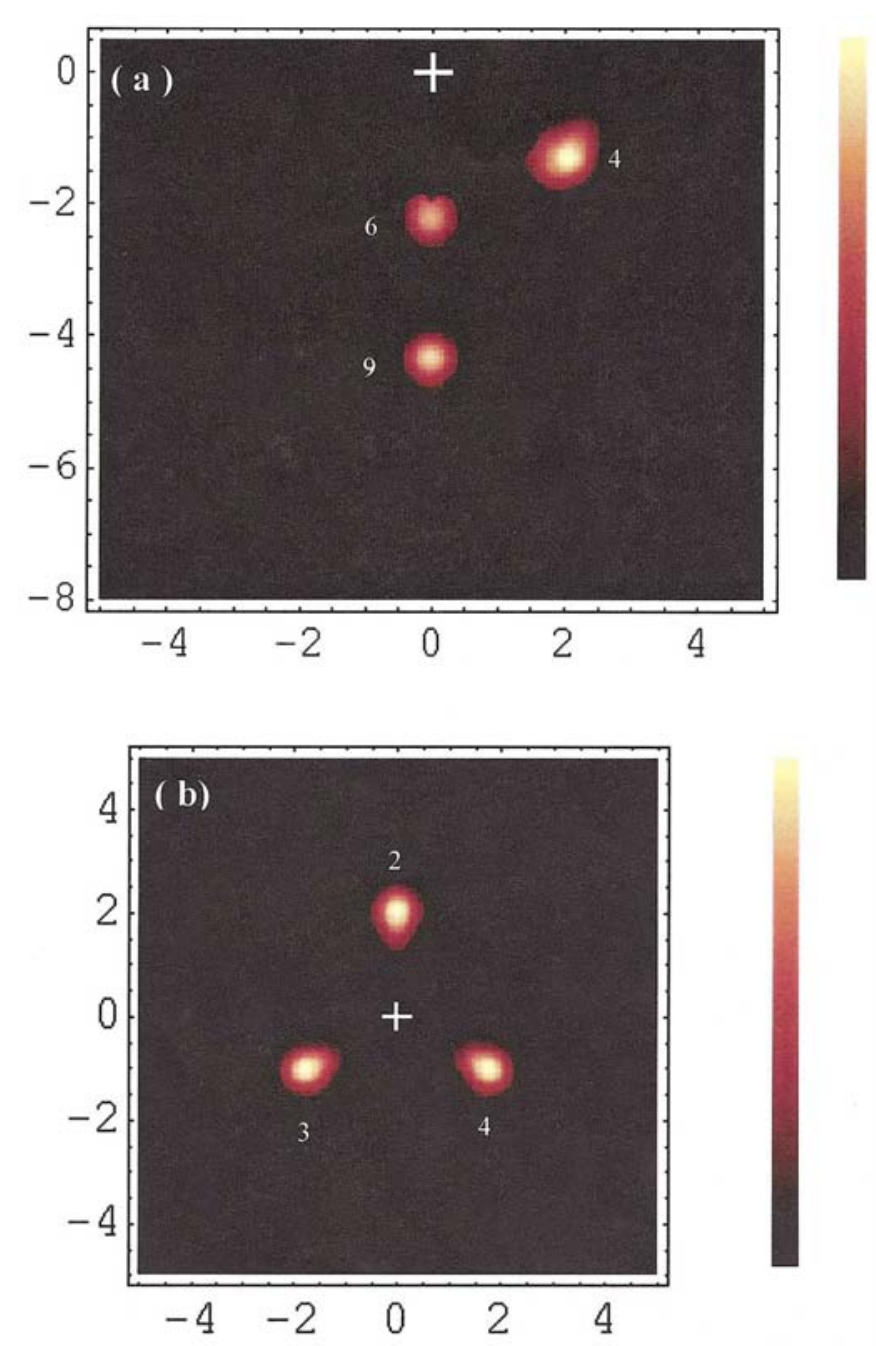

FIG. 4 (color). Atomic images from integral transformation of LEED data using the SHARP kernel with the filter vector $\vec{r}_{14}$. (a) Atoms 4, 6, and 9 in the (101) crystallographic plane relative to the chosen reference atom 1 . (b) Atoms 2, 3, and 4 in a horizontal plane passing through their centers. The unit of the vertical and horizontal scales is $\AA$.

The SHARP kernel uses nonbulk bonds to point to the reference atom. The method is applicable in systems where surface-specific bonds are present, either via the adsorption of foreign atoms or through the rebonding of atoms in a surface reconstruction. Although not demonstrated here, the method should work for systems that do not possess long-range order but do have short-range order by using diffuse LEED data [19]. The method should also work for multidomain structures (e.g., with rotated domains). When a unique bond for one domain is chosen, one should be able to obtain the structure of that domain. In systems with deep multilayer reconstructions, any interatomic bond is surface specific and may be chosen in the SHARP transformation. Finally, in metals with large scattering factors, the number of independent 
data points, i.e., incident angles and energy range, required for a clear image may increase.

We acknowledge the support of RGC Grants No. HKU7117/98P, No. HKU7120/00P, and No. CityU7396/00P.

[1] D. Gabor, Nature (London) 161, 777 (1948); Science 177, 299 (1972).

[2] J. J. Barton, Phys. Rev. Lett. 67, 3106 (1991).

[3] S. Y. Tong, H. Li, and H. Huang, Phys. Rev. Lett. 67, 3102 (1991).

[4] S. Y. Tong, H. Huang, and C. M. Wei, Phys. Rev. B 46, 2452 (1992).

[5] T. G. Tobin, G. D. Waddill, H. Li, and S. Y. Tong, Phys. Rev. Lett. 70, 4150 (1993).

[6] H. Wu, G. J. Lapeyre, H. Huang, and S. Y. Tong, Phys. Rev. Lett. 71, 251 (1993).

[7] C. M. Wei, S.Y. Tong, H. Wedler, M. A. Mendez, and K. Heinz, Phys. Rev. Lett. 72, 2434 (1994).
[8] S. Y. Tong, H. Huang, and X. Q. Guo, Phys. Rev. Lett. 69, 3654 (1992).

[9] T. Gog, P. M. Len, G. Materlik, D. Bahr, C. S. Fadley, and C. Sanchez-Hanke, Phys. Rev. Lett. 76, 3132 (1996).

[10] M. Tegze and G. Faigel, Nature (London) 380, 49 (1996).

[11] D. A. Luh, T. Miller, and T. C. Chiang, Phys. Rev. Lett. 81, 4160 (1998).

[12] K. Reuter, J. Bernhardt, H. Wedler, J. Schardt, U. Starke, and K. Keinz, Phys. Rev. Lett. 79, 4818 (1997).

[13] S. Y. Tong, Adv. Phys. 48, 136 (1999).

[14] W. H. Chen, H. S. Wu, K. B. Ho, B. C. Deng, G. Xu, and S. Y. Tong, Surf. Rev. Lett. 7, 267 (2000).

[15] H.S. Wu and S. Y. Tong, Phys. Rev. Lett. 87, 036101 (2001).

[16] A. L. Patterson, Phys. Rev. 46, 372 (1934).

[17] D. L. Adams and U. Landman, Phys. Rev. B 15, 3775 (1977).

[18] C.Y. Chang, Z. C. Lin, Y. C. Chou, and C. M. Wei, Phys. Rev. Lett. 83, 2580 (1999).

[19] K. Heinz, D. K. Saldin, and J. B. Pendry, Phys. Rev. Lett. 55, 2312 (1985). 